\title{
Changing of the Guard: Interpretive Continuity of the 2005 Strong Interest Inventory
}

\author{
Donna C. Bailey \\ Lisa M. Larson \\ Fred H. Borgen \\ Courtney E. Gasser \\ Iowa State University
}

\begin{abstract}
This study is the first to examine the equivalence of the 2005 Strong Interest Inventory with the 1994 Strong. The authors examine the parallel content scales of the two versions for female and male college students separately $(n=622)$. The scales include the six General Occupational Themes (GOTs), 22 of the 25 Basic Interest Scales (BISs) of the 1994 Strong, and four of the Personal Style Scales (PSSs). The mean differences between the two Strongs were mostly within .5 of a standard deviation (Cohen's $d<.5$ ). There was a pattern of slightly higher means on the 2005 Strong, possibly because of the 2005 Strong standardization sample compares to the 1994 norm group, being more ethnically diverse, less educated, and more representative of the 2000 U.S. Census. The correlations of the 1994 and 2005 content scales were $\geq .85$ for the GOTs and PSSs, except for the Risk Taking/Adventure PSS. The 1994 and 2005 analoged US BIS correlations ranged from .64 to .97 . The effect sizes for sex were comparable across versions.
\end{abstract}

Keywords: vocational interests, interest measurement, Strong Interest Inventory

Often, some of our earliest recollections center around memories of acting out what we wanted to be when we grew up. Who among us is unable to recall afternoons spent as a fire chief, or a ballerina, or even as the president of the United States of America? As we grow older and enter adolescence, our vocational interests became our foremost concern as the focus changes to what we want to do

\footnotetext{
The authors would like to thank David Donnay, Rich Thompson, and CPP, Inc., for their assistance in this project. We would also like to thank Patrick Rottinghaus, Katie Young, Brianne Skahill, and Scott Muhs for their assistance in data collection and data entry. Please send correspondence to Donna C. Bailey, Department of Psychology, Iowa State University, W112 Lagomarcino Hall, Ames, IA 50011-3180; phone: (515) 292-0280; fax: (515) 294-6424; e-mail: dcbailey@iastate.edu.
} 
with our lives. Even as adults, our vocational interests are often the forerunners of many a conversation, with one of the most common questions on meeting someone new being, "So, what do you do?" Vocational interests represent an individual's personal preferences for specific leisure and vocational activities and environments. They are typically encompassed within five domains: personality, motivation or drive, expression of the self-concept, heritability, and influences within the environment such as vicarious learning (Hansen, 1990, 1994).

One of the most influential theories regarding vocational interests is that of John Holland. In his theory, Holland $(1985,1997)$ divides both people and environments into some combination of six vocational domains. These six domains are hexagonally organized, and include realistic (outdoors, mechanical), investigative (science, math), artistic (art, language), social (helping, teaching), enterprising (selling, business) and conventional (details, clerical) (Hansen, 1994). It is not surprising that one of the most influential measures of vocational interests, the Strong Interest Inventory (Strong; Donnay, Thompson, Morris, \& Schaubhut, 2005) incorporates Holland's theory for describing vocational interests and their relation to jobs, people, and environments (Hansen, 1994).

The interest inventory created by E. K. Strong Jr. at Stanford in 1927 has now marked its 80th anniversary. Over the years it has evolved to match new insights about interests and measurement. The original version, the Strong Vocational Interest Blank (Strong, 1927), introduced Strong's landmark vocational scales. These were empirical, contrasted-groups scales, actuarially derived from careful and extensive data collection. Over the decades, revisions of the Strong, especially those led by David Campbell, have been characterized by paradigm shifts toward theoretical organization and content-based homogeneous scales (Campbell \& Borgen, 1999).

The recent revision of the Strong (Donnay et al., 2005) represents a substantial update from the popular 1994 Strong (Harmon, Hansen, Borgen, \& Hammer, 1994). It embodies the publisher's attempts (Consulting Psychology Press, Inc. $[\mathrm{CPP}])$ to capture the evolving occupations, work and leisure activities, and interests of the 21st century. Toward this end, items were added, deleted, and revised. Specifically, the General Occupational Themes (GOTs) were revised, the Basic Interest Scales (BISs) were expanded to 30 with some original scales revised, others combined or omitted, and some new scales added. One additional Personal Style Scale (PSS) was added while other PSS scales were altered. (Note: The Occupational Scales [OSs] were somewhat modified but archival samples from earlier versions were used; thus, they will not be discussed in this article.) Readers can consult the 2005 manual (Donnay et al., 2005) concerning these scales.

Finally, a new normative sample, now referred to as the General Representative Sample (GRS), rather than the General Reference Sample of the 1994 Strong (Harmon et al., 1994), was generated for the 2005 Strong revision (Donnay et al., 2005). The new GRS comprised 2,250 employed adults from the U.S. workforce. It consisted of an equal number of women and men, and participants were elicited exclusively from the Internet (Donnay et al., 2005). 
Given the popularity of the Strong across multiple revisions, it is critical for researchers to generate research using the new Strong for multiple reasons. First, it is important to determine the psychometrics of the 2005 Strong across different populations and settings. Second, it is important to determine the extent to which the 2005 Strong content scales are comparable to the 1994 version for the purpose of test interpretation. This point is imperative because the newer version is already being used across the country and clinicians may assume that the scales provide information identical to the older version. Given the changes in the items based on new items being added, some items being deleted, and some items being edited, this assumption may or may not be empirically grounded. Added to that, the normative group is quite different, with a smaller sample of 2,250 adults who completed the Strong on the Internet, compared to the 18,951 (9,467 women 9,484 men) adults of the 1994 General Reference Sample who completed the Strong as a paper-andpencil test (Harmon et al., 1994). Vocational counselors need data quickly to make empirically informed decisions about the comparability of the 2005 Strong with its 1994 predecessor.

The research concerning the 2005 Strong is understandably scant because of its recent introduction. The 2005 manual (Donnay et al., 2005) presents extensive useful information based on the GRS. Moreover, Gasser, Larson, \& Borgen (2007) recently published a validity study on the 2005 Strong. The authors used a second Internet sample collected by CPP consisting of 1,836 students from across the country who were currently pursuing postsecondary education ranging from associate's degrees to professional degrees. These two sources provide some of the critical information that clinicians need to feel comfortable using the 2005 Strong. However, more research is needed.

The Gasser et al. (2007) study provided initial validity estimates confirming that the 2005 Strong, like the 1994 Strong, is a useful tool in predicting college major. Although establishing the validity of the revised Strong is crucial, it is equally important for users to understand the comparability of the 2005 Strong with the 1994 Strong. For example, a determination must be made as to whether scales with similar names can be interpreted in the same way. Moreover, scales that have been combined, like the 1994 Nature BIS and the 1994 Agriculture BIS, which were combined in the 2005 version, need to be examined. Thus, the first purpose of this study is to provide additional information regarding the equivalence of the 2005 Strong revision to the 1994 Strong.

Specifically toward this first purpose, we examined the equivalency of the following analogous parallel content scales of the two versions: (a) the six GOTs, (b) the 22 BISs, and (c) the four parallel PSSs. (Note: Three 1994 BISs were not included in the 2005 Strong.) We examined equivalency in two ways. First, we compared the means of the 1994 Strong scales and the 2005 Strong scales for women and men separately. Second, we correlated the parallel content scales of the 2005 Strong and the 1994 Strong.

The second purpose of the present study derives from previous research (e.g., Hansen \& Campbell, 1985; Harmon et al., 1994) that has consistently found mean sex differences in some of the content scales of 1994 Strong (as well as in 
earlier versions). True equivalence of the 2005 and 1994 Strongs would suggest similar sex differences between versions. Thus, as a further test of the equivalency between the 2005 and 1994 Strongs, we examined whether sex differences in the 2005 GOTs, BISs, and PSSs were similar in magnitude to the sex differences reported in the 1994 GOTs, BISs, and PSSs.

\section{METHOD}

\section{Participants}

The participants in this study are students in an introductory psychology course at a large upper midwestern university during the fall semester of 2002 and the spring semester of 2003 . There were 355 participants in the fall 2002 data collection pool and 267 participants in the spring 2003 data collection pool for a total of 622 participants. There were 248 men and 374 women with an overall mean age of 19.33 years and a standard deviation of 1.55 . The ethnic breakdown of the sample was as follows: $87.1 \%$ White, non-Hispanic, 2.6\% Hispanic American, 2.7\% Asian American, 3.2\% African American, 2.3\% international students, and 2.1\% of participants marking Other. Participants received course extra credit for their participation, with numerous alternate research options existing for students to gain this course extra credit.

\section{Strong Interest Inventory}

The 1994 Strong Interest Inventory (Harmon et al., 1994) has 317 items and is designed to assess individual interests relative to occupations, people, environments, and leisure activities (Hansen \& Campbell, 1985). The General Reference Sample is 92\% Caucasian and includes 9,467 women and 9,484 men from 200 occupational groups. Each of the three types of content scales (GOTs, BISs, and PSSs) used in this study is described in turn.

The GOTs. The six GOTs are the broadest content scales on the 1994 Strong and align with the RIASEC (realistic, investigative, artistic, social, enterprising, and conventional) typology. This typology provides the underlying theoretical structure for the Strong (Campbell \& Holland, 1972). Harmon et al. (1994) reported Cronbach alphas for each of the GOTs as .90 or higher and all test-retest reliabilities for the GOTs as .80 or higher over a period of 1 to 6 months. Extensive validity data exist regarding the 1994 GOTs (e.g., Donnay \& Borgen, 1996; Harmon et al., 1994; Lattimore \& Borgen, 1999; Olsen, 1996).

The BISs. The $25 \mathrm{BISs}$ of the 1994 Strong were designed as readily interpretable content scales (Campbell \& Borgen, 1999). They were later organized under Holland's hexagon for ease of interpretation (Harmon et al., 1994). The internal consistencies of the $25 \mathrm{BISs}$ are high, with Cronbach alphas ranging 
from .74 for the Agriculture scale ( 6 items) to .94 for the Mechanical Activities scale (21 items; Harmon et al., 1994). These same researchers reported 3 to 6 month test-retest correlations for the BISs ranging from .80 for Culinary Arts and Teaching to .94 for Athletics. Extensive validity information is available on the 1994 BISs (e.g., Donnay \& Borgen, 1996; Hansen, 1986; Harmon et al., 1994; Lattimore \& Borgen, 1999).

The PSSs. The four PSSs include Work Style, Learning Environment, Leadership Style, and Risk Taking/Adventure. The PSSs are moderately intercorrelated with the highest correlation falling between the Leadership Style and Work Style scale ( $r s=.61, .52$ for men and women, respectively). The Work Style and Learning Environment PSSs show nominal correlations (Harmon et al., 1994). Internal consistency estimates range from .78 for Risk Taking/Adventure to .91 for Work Style (Harmon et al., 1994). Three to 6 month test-retest reliabilities range from .81 for Leadership Style to .92 for Work Style (Harmon et al., 1994). The validity of the PSSs is well established (e.g., Harmon et al., 1994).

\section{Strong Interest Inventory}

The 2005 Strong Interest Inventory (Donnay et al., 2005) contains 291 items and three types of content scales: six General Occupational Themes, 30 Basic Interest Scales, and five Personal Style Scales. The adult GRS consists of an equal number of women and men $(N=2,250)$ garnered from the U.S. workforce. The mean age of the respondents was 35 ; they averaged 9 years of experience in their respective occupations and reported working a mean of $41 \mathrm{hr}$ per week (Donnay et al., 2005). Additionally, the new GRS represents the diversity of the U.S. workforce, consisting of 373 different occupations (Donnay et al., 2005).

Two notable differences should be noted in the 2005 GRS in comparison to the 1994 General Reference Sample. First, the 2005 GRS is more ethnically diverse than the 1994 standardization sample. The 2005 male GRS represents the U.S. racial and ethnic distribution found in the 2000 Census (24\%), whereas the female GRS overrepresents the U.S. racial and ethnic distribution in the 2000 Census (32\%; Donnay et al., 2005). Second, the 2005 GRS is less educated than the 1994 General Reference Sample. Specifically, the proportion of respondents who did not have a bachelor's degree was $43 \%$ in the 2005 GRS, in contrast to $23 \%$ of women and $18 \%$ of men in the 1994 standardization sample. Likewise, only $3.4 \%$ of the 2005 GRS had a PhD or professional degree, in contrast to $16 \%$ of the women and $21 \%$ of the men in the 1994 standardization sample (Donnay et al., 2005).

GOTs. To retain its interpretative validity, the developers of the new Strong retained the hexagonal structure of the GOTs based on Holland's (1985) theory. Moreover, any differences observed in the 2005 GOTs most likely reflect changes made to the BISs. The main effect of the BIS changes was to broaden and expand the GOTs to include technological advances in the workplace. For 
example, the Conventional theme was broadened and now includes items measuring computer programming and working with software (Donnay et al., 2005). This expansion of the GOTs served to increase the internal consistency reliability of five of the themes (Realistic remained constant at .93), with all six themes possessing Cronbach alpha's of .91 or higher (Donnay et al., 2005). The Cronbach alphas for the 2005 Strong in the current sample were computed to examine the internal consistency of the GOTs as shown in Table 1. The alpha's of the GOTs demonstrated high internal consistency and ranged from .90 (Enterprising) to .93 (Artistic and Conventional).

BISs. The BISs were increased from 25 in the 1994 Strong to 30 in the 2005 Strong. In all, 4 scales were dropped (i.e., Computer Activities, Art, Merchandising, and Data Management), 10 new scales were added (i.e., Computer Hardware and Electronics, Protective Services, Research, Human Resources and Training, Social Sciences, Marketing and Advertising, Entrepreneurship, Taxes and Accounting, Programming and Information Systems, and Finance and Investing), and three scales were reconstructed (i.e., the 1994 Nature and 1994 Agriculture BISs are now the 2005 Nature and Agriculture BIS; the 1994 Law/Politics and 1994 Public Speaking BISs are now the 2005 Law and 2005 Politics and Public Speaking BISs). The remaining BISs were revised and updated (Donnay et al., 2005). Additionally, the number of items per scale was reduced from 5 to 21 items per BIS in 1994 to 6 to 12 items per BIS in 2005 (Donnay et al., 2005). The overall internal consistency remained unchanged for the 2005 Strong BISs with a median Cronbach's alpha of .87 (Donnay et al., 2005). As illustrated in Table 1, the 2005 BIS Cronbach alphas for the current sample ranged from .76 (Management) to .91 (Mechanics and Construction, Religion and Spirituality, Sales, and Law).

PSSs. The four PSSs first introduced in the 1994 Strong remained relatively unchanged in the 2005 version. The Risk Taking/Adventure scale is an exception. It was broadened from focusing primarily on physical risk taking in the 1994 Strong to also encompass financial and emotional risk taking in the 2005 Strong (Donnay et al., 2005). A second change with the 2005 Strong PSSs is the addition of the Team Orientation scale. As with the other PSSs, Team Orientation is measured on two poles. Its two poles range from preferring to accomplish tasks independently to preferring to accomplish tasks collectively (Donnay et al., 2005). The PSSs were normed on the GRS $(N=2,250)$. The intercorrelations for women and men between scales were acceptable. They ranged from .13 (.02 for men) between the Work Style scale and the Learning Environment scale to .55 (.54 for men) between the Leadership Style scale and the Team Orientation scale (Donnay et al., 2005). The alphas reported by Donnay and colleagues ranged from .82 for the Risk Taking scale to .87 for the Leadership Style scale. As shown in Table 1, the alphas in this sample ranged from .76 for the Learning Environment scale to .87 for the Work Style scale. 


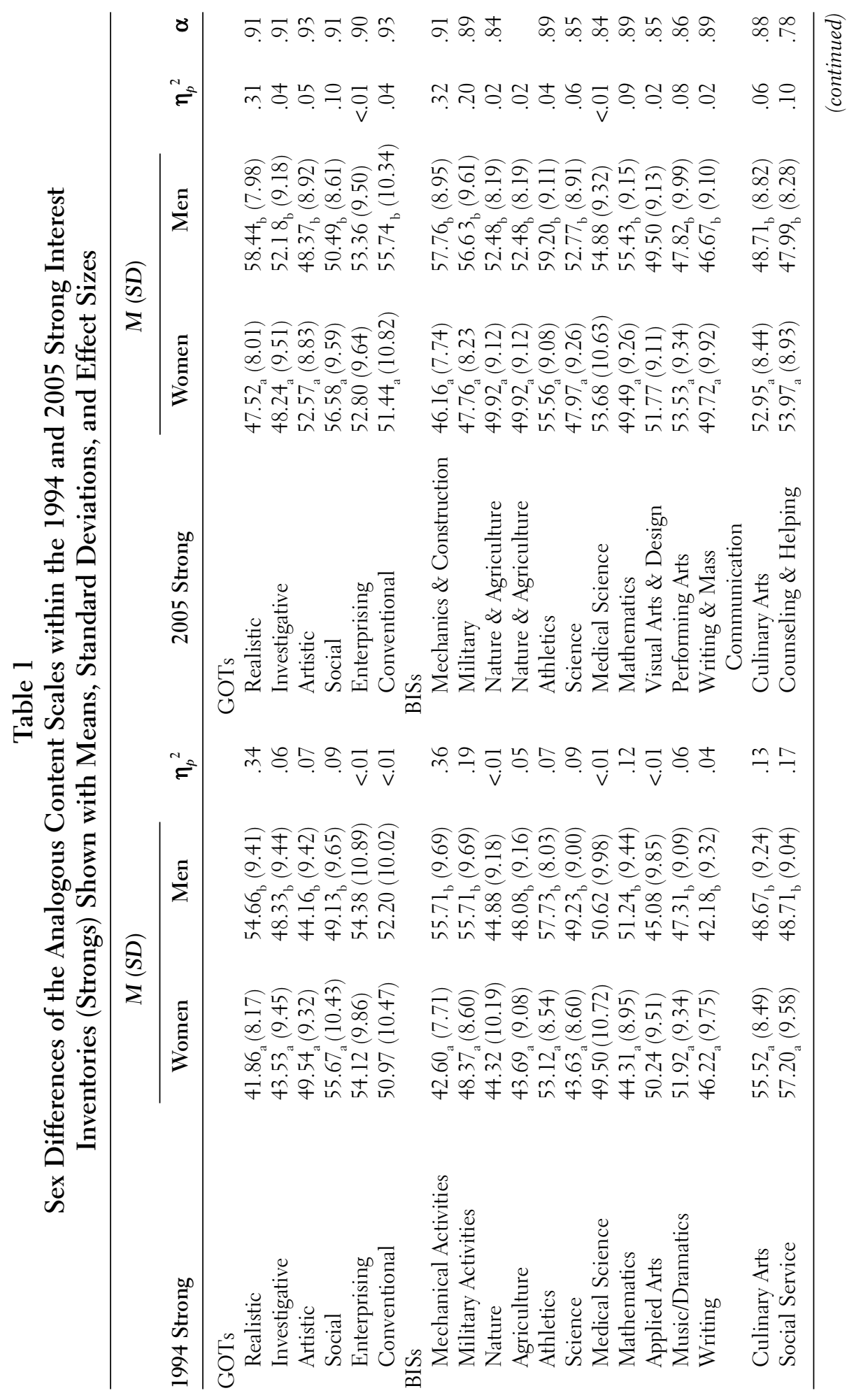




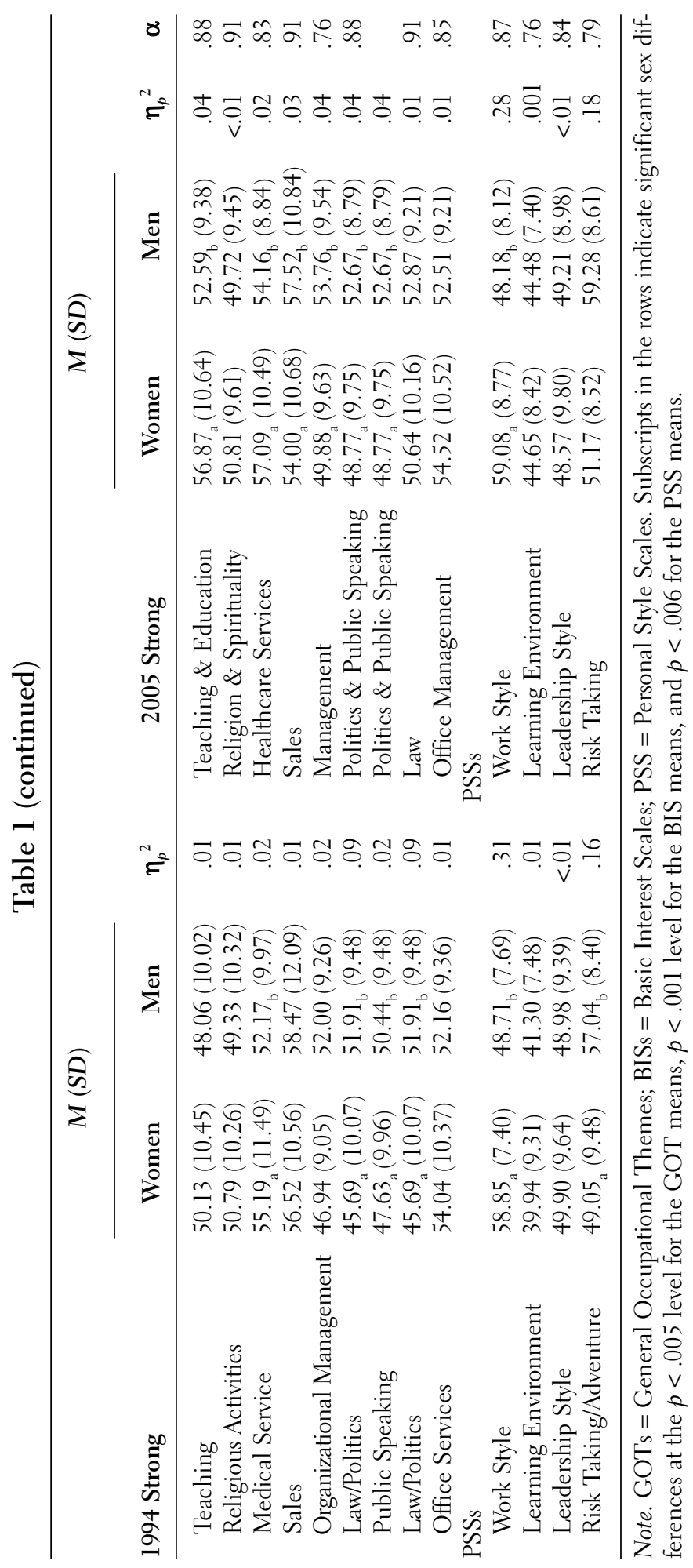




\section{Procedures}

All participants were volunteers selected from a mass-testing session of introductory psychology students participating for course extra credit in the fall of 2002 and spring of 2003. During a $2.5 \mathrm{hr}$ data collection session, students completed in a counter-balanced order the Research Version of the 2005 Strong, the Multidimensional Personality Questionnaire (MPQ; Tellegen, 1982, 2000), and the Expanded Skills Confidence Inventory (ESCI; Betz et al., 2003). (Note: The 2005 Strong booklets were not yet released during the periods of active data collection. The Research Version contained 428 items including the 317 items from the 1994 Strong, 78 new items from the 2005 Strong, and an additional 33 items that were ultimately not included in the revised Strong. All items were answered on the new 5-point (from strongly like to strongly dislike) response format of the 2005 Strong. The MPQ and ESCI were not a part of this study and so are not discussed further. Because of the length of the test administration, order was tested as an independent variable with each of the content scales for both versions as dependent variables for both women and men. A conservative $p$ value was set given the number of tests. Order exerted a nominal effect given that only 4 of 152 comparisons were significant. We also examined whether time of data collection was significant. The scores did not differ across semesters.

\section{RESULTS}

\section{Purpose 1: Equivalency of the Analogous Content Scales of the 2005 and 1994 Strongs}

Comparison of the means of the analogous content scales of the 2005 and 1994 Strongs by sex. In accordance with the first purpose of this study, to determine if significant differences exist between the means of the parallel content scales of the 1994 and 2005 Strongs, two sets of paired sample $t$ tests were conducted. The paired-sample $t$ tests were computed separately for each sex in which year was the independent variable (1994 versus 2005) and the dependent variables were the analogous content scales. A Bonferroni adjustment was used to control for multiple tests (GOTs: $p s<.005$; BISs: $p s<.001$; PSSs: $p s<.006$ ).

As can be seen in Tables 2 and 3, for both sexes, the 2005 GOT means were significantly higher than the 1994 means, with two exceptions. First, the 2005 Enterprising GOT mean was significantly lower than the 1994 Enterprising GOT mean for both women and men. Second, the 1994 and 2005 Conventional GOT means were not significantly different for women, as shown in Table 2 . With respect to the magnitude of the mean differences, Cohen's $d$ was used with positive numbers reflecting higher means on the 2005 Strong. Cohen's $d$ is defined as the difference between independent means expressed in units of the within-population standard deviation; $d s$ equal to or greater than .5 and less than .8 are considered 
Table 2

Comparison of Means, Standard Deviations, and Correlations of the Analogous Content Scales (GOTs, BISs, and PSSs) of the 1994 and 2005 Strong Interest Inventories for Women $(n=374)$

\begin{tabular}{|c|c|c|c|c|}
\hline & \multicolumn{4}{|c|}{ Women } \\
\hline & $\begin{array}{l}1994 \text { Strong } \\
\quad M(S D)\end{array}$ & $\begin{array}{l}2005 \text { Strong } \\
\quad M(S D)\end{array}$ & $\begin{array}{l}\text { 1994/2005 } \\
\text { Strongs } \\
\text { Cohen's d }\end{array}$ & $\begin{array}{c}1994 / 2005 \\
\text { Strongs } r\end{array}$ \\
\hline \multicolumn{5}{|l|}{1994 (2005) GOTs } \\
\hline Realistic & $41.86(8.17)$ & $47.52(8.01)$ & .70 & .85 \\
\hline Investigative & $43.53(9.45)$ & $48.24(9.51)$ & .50 & .94 \\
\hline Artistic & $49.54(9.32)$ & $52.57(8.83)$ & .33 & .96 \\
\hline Social & $55.67(10.43)$ & $56.58(9.59)$ & .09 & .92 \\
\hline Enterprising & $54.12(9.86)$ & $52.80(9.64)$ & -.14 & .91 \\
\hline Conventional & $50.97(10.47)$ & $51.44(10.82)$ & .06 & .89 \\
\hline \multicolumn{5}{|l|}{1994 (2005) BISs } \\
\hline $\begin{array}{l}\text { Mechanical Activities } \\
\text { (Mechanics \& Construction) }\end{array}$ & $42.60(7.71)$ & $46.16(7.74)$ & .46 & .88 \\
\hline Military Activities (Military) & $48.37(8.60)$ & $47.76(8.23)$ & -.07 & .85 \\
\hline Nature (Nature \& Agriculture) & $44.32(10.19)$ & $49.92(9.12)$ & .58 & .86 \\
\hline Agriculture (Nature \& Agriculture) & $43.69(9.08)$ & $49.92(9.12)$ & .68 & .68 \\
\hline Athletics & $53.12(8.54)$ & $55.56(9.08)$ & .28 & .93 \\
\hline Science & $43.63(8.60)$ & $47.97(9.26)$ & .49 & .90 \\
\hline Medical Science & $49.50(10.72)$ & $53.68(10.63)$ & .39 & .87 \\
\hline Mathematics & $44.31(8.95)$ & $49.49(9.26)$ & .57 & .92 \\
\hline Applied Arts (Visual Arts \& Design) & $50.24(9.51)$ & $51.77(9.11)$ & .16 & .88 \\
\hline Music/Dramatics (Performing Arts) & $51.92(9.34)$ & $53.53(9.34)$ & .17 & .91 \\
\hline $\begin{array}{l}\text { Writing (Writing \& Mass } \\
\text { Communication) }\end{array}$ & $46.22(9.75)$ & $49.72(9.92)$ & .36 & .90 \\
\hline Culinary Arts & $55.52(8.49)$ & $52.95(8.44)$ & -.30 & .83 \\
\hline $\begin{array}{l}\text { Social Service (Counseling \& } \\
\text { Helping) }\end{array}$ & $57.20(9.58)$ & $53.97(8.93)$ & -.35 & .78 \\
\hline Teaching (Teaching \& Education) & $50.13(10.45)$ & $56.87(10.64)$ & .64 & .91 \\
\hline $\begin{array}{l}\text { Religious Activities (Religion \& } \\
\text { Spirituality) }\end{array}$ & $50.79(10.26)$ & $50.81(9.61)$ & .01 & .93 \\
\hline $\begin{array}{l}\text { Medical Service (Healthcare } \\
\text { Services) }\end{array}$ & $55.19(11.49)$ & $57.09(10.49)$ & .17 & .93 \\
\hline Sales & $56.52(10.56)$ & $54.00(10.68)$ & -.24 & .86 \\
\hline $\begin{array}{l}\text { Organizational Management } \\
\text { (Management) }\end{array}$ & $46.94(9.05)$ & $49.88(9.63)$ & .31 & .85 \\
\hline $\begin{array}{l}\text { Law/Politics (Politics \& Public } \\
\text { Speaking) }\end{array}$ & $45.69(10.07)$ & $48.77(9.75)$ & .31 & .86 \\
\hline $\begin{array}{l}\text { Public Speaking (Politics \& Public } \\
\text { Speaking) }\end{array}$ & $47.63(9.96)$ & $48.77(9.75)$ & .12 & .89 \\
\hline Law/Politics (Law) & $45.69(10.07)$ & $50.64(10.16)$ & .31 & .79 \\
\hline $\begin{array}{l}\text { Office Services (Office } \\
\text { Management) }\end{array}$ & $54.04(10.37)$ & $54.52(10.52)$ & .05 & .87 \\
\hline
\end{tabular}


Table 2 (continued)

\begin{tabular}{lcccc}
\hline & \multicolumn{4}{c}{ Women } \\
\cline { 2 - 5 } & $\begin{array}{c}\text { 1994 Strong } \\
\mathbf{M}(\mathrm{SD})\end{array}$ & $\begin{array}{c}\text { 2005 Strong } \\
\mathbf{M}(\mathrm{SD})\end{array}$ & $\begin{array}{c}\text { Strongs } \\
\text { Cohen's } \boldsymbol{d}\end{array}$ & $\begin{array}{c}\text { 1994/2005 } \\
\text { Strongs } \boldsymbol{r}\end{array}$ \\
\hline 1994 (2005) PSSs & & & & \\
Work Style & $58.85(7.40)$ & $59.08(8.77)$ & .03 & .92 \\
Learning Environment & $39.94(9.31)$ & $44.65(8.42)$ & .53 & .88 \\
Leadership Style & $49.90(9.64)$ & $48.57(9.80)$ & -.14 & .95 \\
Risk Taking/Adventure & $49.05(9.48)$ & $51.17(8.52)$ & .24 & .74 \\
$\quad$ (Risk Taking) & & & & \\
\hline
\end{tabular}

Note . GOTs $=$ General Occupational Themes; BISs $=$ Basic Interest Scales; PSSs $=$ Personal Style Scales. The names for the 2005 Strong scales are identical to the 1994 Strong scales unless otherwise noted in the parentheses. Means that are in bold in the same rows indicate significant mean differences in the 1994 and 2005 Strong scales at the $p<.005$ level for the GOTs, at the $p<.001$ level for the BISs, and the $p<.006$ level for the PSSs. Positive numbers for Cohen's $d$ reflect higher means on the 2005 Strong scales.

medium effects (Cohen, 1988). As presented in Tables 2 and 3, there were only two medium effect sizes, namely the Realistic and Investigative GOT means for women $(d s=.70, .50$, respectively). The remainder of significant effects was small.

As seen in Tables 2 and 3, most of the 2005 BIS means for men and women were significantly higher than the 1994 BIS means at the $p<.001$ level. The exceptions were as follows. Three 1994 BIS means for women were higher than their corresponding 2005 BIS means: Culinary Arts, Social Science (2005: Counseling and Helping), and Sales. The following BIS means were not significantly different: Religious Activities (2005: Religion and Spirituality) BISs and the Office Services (2005: Office Management) BISs for both sexes, the Military Activities (2005: Military) BISs for women, and six BIS means for men, including the Music/ Dramatics (2005: Performing Arts) BISs, the Culinary Arts BISs, the Social Service (2005: Counseling and Helping) BISs, the Sales BISs, the Law/Politics (2005 Politics/Public Speaking) BISs, and the Law/Politics (2005 Law) BISs.

Several 1994/2005 mean differences between BISs were large enough to constitute at least a medium effect of $d=.5$, as shown in Tables 2 and 3. First, for women and men respectively, the 2005 Nature and Agriculture BIS means were higher than the 1994 Nature BIS means $(d s=.58, .87)$ and the 1994 Agriculture BIS means $(d s=.68, .51)$. Second, for women only, their 2005 Mathematics BIS mean was higher than their 1994 Mathematics BIS mean $(d=.57)$, and their 2005 Teaching and Education BIS mean was higher than their corresponding 1994 BIS mean $(d=.64)$.

Again, as shown in Tables 2 and 3, the majority of the means for the 1994 and 2005 PSSs were significantly different at the $p<.006$ level. Two exceptions were 
Table 3

Comparison of Means, Standard Deviations, and Correlations of the Analogous Content Scales (GOTs, BISs, and PSSs) of the 1994 and 2005 Strong Interest Inventories for Men $(n=248)$

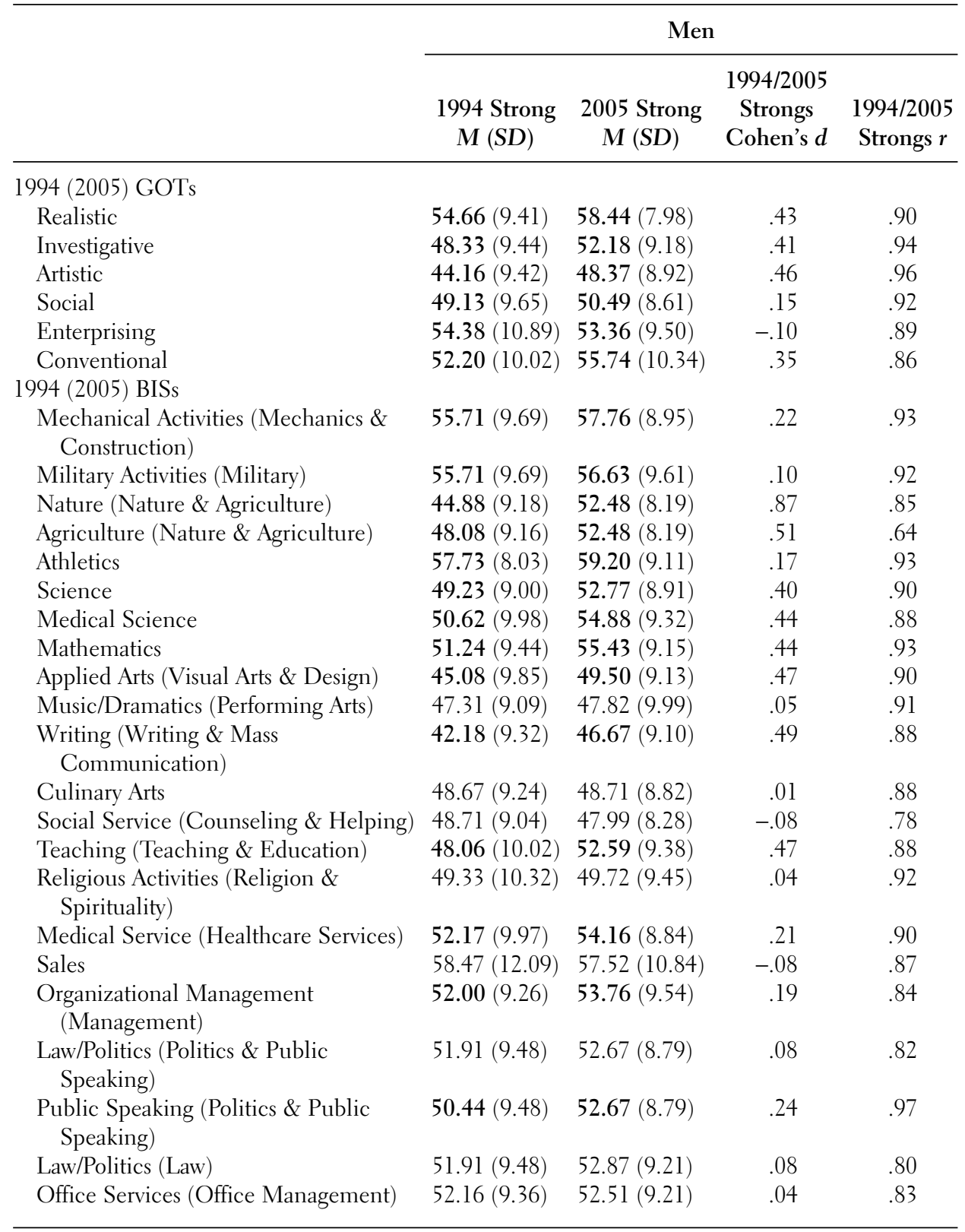


Table 3 (continued)

\begin{tabular}{|c|c|c|c|c|}
\hline & \multicolumn{4}{|c|}{ Men } \\
\hline & $\begin{array}{c}1994 \text { Strong } \\
M(S D)\end{array}$ & $\begin{array}{c}2005 \text { Strong } \\
M(\mathrm{SD})\end{array}$ & $\begin{array}{l}\text { 1994/2005 } \\
\text { Strongs } \\
\text { Cohen's d }\end{array}$ & $\begin{array}{c}1994 / 2005 \\
\text { Strongs } r\end{array}$ \\
\hline \multicolumn{5}{|l|}{1994 (2005) PSSs } \\
\hline Work Style & $48.71(7.69)$ & $48.18(8.12)$ & -.07 & .94 \\
\hline Learning Environment & $41.30(7.48)$ & $44.48(7.40)$ & .43 & .79 \\
\hline Leadership Style & $48.98(9.39)$ & $49.21(8.98)$ & .03 & .94 \\
\hline Risk Taking/Adventure (Risk Taking) & $57.04(8.40)$ & $59.28(8.61)$ & .26 & .71 \\
\hline
\end{tabular}

Note . GOTs = General Occupational Themes; BISs = Basic Interest Scales; PSSs = Personal Style Scales. The names for the 2005 Strong scales are identical to the 1994 Strong scales unless otherwise noted in the parentheses. Means that are in bold in the same rows indicate significant mean differences in the 1994 and 2005 Strong scales at the $p<.005$ level for the GOTs, at the $p<.001$ level for the BISs, and the $p<.006$ level for the PSSs. Positive numbers for Cohen's $d$ reflect higher means on the 2005 Strong scales.

the Work Style PSS for women and the Leadership Style PSS for men. As seen in Tables 2 and 3, the 2005 Learning Environment mean and the 2005 Risk Taking mean were significantly higher than their analogous 1994 PSS means for both women and men. The 2005 Leadership Style PSS was significantly lower than its 1994 counterpart only for the women. However, the magnitudes of the differences in the PSS means were small. Only the women's Learning Environment PSS mean in 2005 when compared to 1994 was meaningfully higher, with a Cohen's $d$ of .53 .

Correlating the 2005 and 1994 Strong Interest Inventories within sex. Also in alignment with the first purpose of the present study, Pearson product-moment correlations were used to test for the equivalency of the analogous content scales of the 1994 and 2005 Strongs. Table 2 presents the correlations between all analogous Strong content scales for women, and Table 3 presents the correlations between all analogous Strong content scales for men. The 1994/2005 correlations between the parallel GOTs ranged from .85 on the Realistic GOT for women to .96 for both women and men on the Artistic GOTs. These strong positive correlations provide compelling evidence for the equivalency of the GOTs across versions.

The correlations of the 22 analogous BISs of the 1994 and 2005 Strongs are also shown in Tables 2 and 3. As shown in Table 2, for women, the highest 1994/2005 BIS correlations were Athletics, Religious Activities (2005: Religion \& Spirituality), Medical Service (2005: Healthcare Services) $(r s=.93)$, and Mathematics (.92). The lowest correlations for women were Agriculture (2005: Nature and Agriculture) $(r=.68)$, Social Service (2005: Counseling and Helping) $(r=$ .78), and Law/Politics (2005: Law) $(r=.79)$. As shown in Table 3, the highest correlations for men were Public Speaking (2005: Politics and Public Speaking) 
$(r=.97)$, Mechanical Activities (2005: Mechanics \& Construction), and Athletics, and Mathematics $(r s=.93)$. The lowest correlations for men were Agriculture (2005: Nature and Agriculture; $r=.64)$, Social Service (2005: Counseling and Helping, $r=.78$ ), and Law/Politics (2005: Law; $r=.80$ ).

For the PSSs, the Work Style PSS and the Leadership Style PSS was highly correlated for both women and men. As shown in Tables 2 and 3, these correlations ranged from .92 to .95. The Risk Taking/Adventure (2005: Risk Taking) PSS was correlated .74 for women and .71 for men. The Learning Environment correlation was lower for men (.79) than for women (.88).

\section{Purpose 2: Equivalency of the Sex Differences between the 2005 and 1994 Strongs}

Comparison of the pattern of mean sex differences within the 2005 Strong versus the 1994 Strong. In accordance with the second purpose of the present study, univariate analysis of variances (ANOVAs) were conducted on the content scales of the 1994 and 2005 Strong Interest Inventories. Sex was the independent variable and each Strong content scale was the dependent variable. Each univariate ANOVA was tested at the Bonferroni adjusted level of $p<.005$ for the GOTs, $p<.001$ for the BISs, and $p<.006$ for the PSSs. Table 1 presents the means by sex for each content scale and indicates which sex differences were significant by the use of a subscript beside the female mean. For example, there is a subscript beside the Realistic GOT mean for women for both versions of the Strong in Table 1 to indicate that women scored significantly lower than men.

As seen in Table 1, for both the 1994 and the 2005 Strongs, men had higher means on the Realistic and Investigative GOTs, whereas women had higher means on the Artistic and Social GOTs ( $p s<.005)$. Neither version of the Strong showed sex differences on the Enterprising GOT. Finally, men compared to women scored significantly higher on the 2005 Conventional GOT only. In essence, five of the six GOTs showed a similar pattern of sex differences.

To compare the magnitude of the mean sex differences across the two versions, partial eta squared values were computed as effect sizes and are listed in Table 1. The partial eta squared value represents the proportion of variance in one variable that is explained by the second variable. Cohen (1988) considers .06 to .14 to be a medium effect. The partial eta squared values were mostly similar across the two versions. As expected, strong effects were found for both the 1994 and 2005 Realistic GOTs $(.34, .31)$, whereas medium effects were found for the 1994 and 2005 Social GOTs $(.09, .10)$, the 1994 Investigative GOT (.06), and the 1994 Artistic GOT (.07). The partial eta squared values for the remaining GOT significant mean differences ranged from .04 to .05 indicating small effect sizes.

The BIS sex differences within each version of the Strong are also presented in Table 1. In 17 analogous pairs, a similar pattern emerged across both the 1994 and the 2005 BISs. That is, 13 sex differences ( $p$ < <.001) emerged across the 1994 BISs 
and the corresponding 2005 BISs. Specifically, as shown in Table 1, women scored higher on the following BISs across both versions: Music/Dramatics (2005: Performing Arts), Writing (2005: Writing and Mass Communication), Culinary Arts, Social Service (2005: Counseling and Helping), and Medical Service (2005: Healthcare Services). Men scored higher on Mechanical Activities (2005: Mechanics \& Construction), Military Activities (2005: Military), Agriculture (2005: Nature and Agriculture), Athletics, Science, Mathematics, Law/Politics (2005: Politics/Public Speaking), Public Speaking (2005: Politics/Public Speaking). Moreover, four additional parallel BISs (i.e., Medical Science, Applied Arts/[2005: Visual Arts and Design], Religious Activities [2005: Religion and Spirituality], and Office Services [2005: Office Management]) did not reveal sex differences across either version of the Strong.

Five analogous scales demonstrated a different pattern across the two versions as seen in Table 1. In all five instances, the means for women and men were significant at the $p<.001$ level in one version but not in the second. Specifically, men scored significantly higher than women on the 2005 Nature BIS (compared to the 1994 Sales BIS) and Agriculture BIS (compared to the 1994 Nature BIS), the 2005 Sales BIS, the 2005 Management BIS (compared to the 1994 Organizational Management BIS), and the 1994 Law/Politics BIS (compared to the 2005 Law BIS); whereas the women scored significantly higher than the men on the 2005 Teaching BIS (compared to the 1994 Teaching BIS). However, the effect sizes of these mean differences were small, ranging from $\eta_{p}^{2}=.02$ to .04 .

When the partial eta squared values were examined, comparable effect sizes for sex for the 1994 and the 2005 BISs were apparent, as seen in Table 1. For both versions, the largest effect sizes were the Mechanical Activities (2005: Mechanics \& Construction) BISs with $\eta_{p}^{2}=.36, .32$, respectively; the Military Activities (2005: Military) BISs with $\eta_{p}^{2}=.19$, .20, respectively; and the Social Service (2005: Counseling and Helping) BISs with $\eta_{p}^{2}=.17, .10$, respectively. These BIS results suggest similar differential interpretations based on sex for both versions. Medium effect sizes $\left(.13 \geq \eta_{p}^{2} \geq .06\right)$ were observed for four of the 1994-to-2005 parallel BISs (i.e., Science, Mathematics, Music/Dramatics [2005: Performing Arts], and Culinary Arts). Finally, small effect sizes $\left(\eta_{p}{ }^{2} \leq .05\right)$ were observed for five of the parallel 1994/2005 BISs (i.e., Agriculture [2005: Nature and Agriculture], Athletics, Writing [2005: Writing \& Mass Communication], Medical Service [2005: Healthcare Services], and Law/Politics [2005: Politics \& Public Speaking]).

Finally, the PSS means by sex are presented in Table 1. Specifically, women had significantly higher means on both the 1994 and 2005 Work Style PSSs, whereas the men had significantly higher means on the 1994 Risk Taking/Adventure and the 2005 Risk Taking PSSs $(p s<.006)$. The Learning Environment and Leadership Style PSS means for both the 1994 and 2005 Strongs did not differ by sex at the $p<$ .006 level. Additionally, effect sizes were parallel for both versions with the 1994 and 2005 Work Style and Risk Taking/Adventure (2005: Risk Taking) scales having large effect sizes (Work Style: $\eta_{p}^{2}=.31$, .28; Risk Taking: $\eta_{p}^{2}=.16$; .18). 


\section{DISCUSSION}

\section{Equivalency of the 2005 and 1994 Strongs}

GOTs. Based on this sample, in general, the 2005 GOTs mostly seem comparable to the 1994 GOTs. The parallel content scales correlated highly with one another, and the means of the parallel scales were mostly within .5 of a standard deviation. Across both versions, men showed substantially more realistic interests, whereas women showed more social interests. The magnitude of those sex differences was comparable. Enterprising interests did not differ by sex for either version. Despite their equivalence, some differences need to be noted between the two versions in this sample. First, women expressed more realistic and investigative interests $(d s \geq .5)$ on the 2005 Strong compared to the 1994 Strong. This expression of increased interest may be due to the 2005 GRS being more ethnically diverse and less educated than the 1994 General Reference Sample. These differences do need to be followed up in future studies to determine their significance. However, in general, it seems that the 2005 GOTs in this study were generally equivalent to their 1994 predecessors.

BISs. The majority of the evidence points to the 2005 analogous BISs as being mostly equivalent to their respective 1994 BISs. Of the 22 1994-to-2005 analogous BIS Pearson product-moment correlations for the female sample and the male sample, 18 and 16 respectively yielded correlations at or above .85 , as shown by Tables 2 and 3. Moreover, all but six of the means across the two parallel versions for the female and male samples yielded effect sizes in which Cohen's $d$ was $<$.5. Finally, on both versions of the Strong, for 17 of the 22 parallel BISs, the pattern of sex differences was comparable, and for the remaining $5 \mathrm{BISs}$, the effect sizes were small, with $\eta_{p}{ }^{2} \leq .04$. These results are impressive and suggest support for the achievement of maintaining the integrity and essence of the 1994 Strong while simultaneously attempting to update the 2005 Strong to reflect the changing nature of the work force. However, some differences emerged between the two versions that deserve a closer examination in future studies.

First, we noted a pattern in our sample that the women's T-scores, and to a lesser extent, the men's T-scores, were higher than their 1994 T-scores. For example, as shown in Table 2, all but four of the women's 2005 BIS means were higher than their 1994 BIS means. We suspect the reason may be that the 2005 GRS was more ethnically diverse and less educated than the 1994 standardization sample. Future researchers need to continue to examine whether this pattern is consistent with other research.

Second, we found contrary evidence to the equivalence of the merging of the Nature and Agriculture BISs into one BIS in the 2005 Strong. The 1994 Agriculture and 2005 Nature and Agriculture scales (for both sexes) yielded the lowest correlations ( $r s=.68$ for women and .64 for men). Interestingly, the 1994 
Nature BIS correlated more with the 2005 Nature and Agriculture scale $(r s=.86$ for women and .85 for men). Given the relative decrease in strength of these correlations, the decision to combine these scales in the 2005 Strong becomes uncertain.

Further evidence of this uncertainty is provided in Staggs, Larson, and Borgen (2007), who conducted a meta-analysis of the convergence of interests and personality using the 1985 and 1994 Strongs and the MPQ (Tellegen, 1982, 2000). In this study, the MPQ Absorption scale, the tendency to become engrossed in external stimuli, was positively correlated with the 1994 Nature BIS but not the 1994 Agriculture BIS. Conversely, the MPQ Harmavoidance scale, the tendency to enjoy dangerous activities and to seek them out, was related to the 1994 Agriculture BIS but not the 1994 Nature BIS. This evidence suggests that CPP may have collapsed two interests that are distinct. It is also important to note that because of proprietary restrictions by CPP, we were not able to examine scale comparability as to the item composition.

Third, the 1994 Teaching BIS and the 1994 Mathematics BIS correlated highly with their analogous 2005 BISs but the students, particularly the women, expressed stronger interests on the 2005 Strong, as shown in Table 3. This may be because of item revisions or it may be because of the different reference groups for the older and newer versions of the Strong. It could also be unique to this sample; thus, more researchers need to examine these particular BISs.

Fourth, the 1994 Social Service BIS and the 1994 Law/Politics BIS had similar means to their 2005 parallel BISs (2005 Counseling and Helping BIS and 2005 Law BIS), but they correlated less than the other parallel scales, with correlations ranging from .78 to .80 . The 1994 Law/Politics BIS was split into the 2005 Politics and Public Speaking BIS and the 2005 Law BIS. The lower correlation could reflect the splitting of the items from one scale to two scales. These results need to be replicated in other samples.

PSSs. According to this sample, the majority of analogous PSSs between the 2005 and 1994 Strongs were equivalent. The mean differences between the 1994 and 2005 analogous PSSs were small with the exception of the Learning Environment PSS for women. The women's Learning Environment scale 2005 T-score mean, compared to their 1994 T-score mean, was substantially higher. One explanation might be the differences between the 2005 and the 1994 standardization samples with respect to diversity and education.

The Pearson product-moment correlations of the analogous PSSs were quite high with a few exceptions. The most substantive difference to emerge in the PSSs is that the 1994 Risk Taking/Adventure PSS correlate less with the 2005 Risk Taking PSS than anticipated $(r s=.74, .71$ for women and men, respectively). The lower correlation is most likely because of the change in content from the 1994 version that emphasized physical risk to include financial and emotional risk in the 2005 version. Other research has suggested that financial risk taking and physical risk taking are not the same (e.g., Staggs et al., 2007). 
The strong positive relations found between the overwhelming majority of the analogous content scales of the1994 and the 2005 Strong is arguably the most important finding in this study. Determining how well the content scales across the 1994 and 2005 Strongs relate ensures that the interpretation of the scales for the 2005 version closely approximate the interpretation of the scales using the 1994 version. However, those scales with lower than expected correlations should be examined further.

\section{GRS}

In examining the Cohen's $d s$ in Tables 2 and 3, it seems that in general participants' standard scores on their 2005 Strongs were higher than their 1994 Strongs. The large and medium effects have been noted in the article and can be seen in Tables 2 and 3. One explanation may be differences in the normative group. Besides the obvious differences of the 2005 GRS being a smaller, Internet sample compared to a much larger, traditional paper-and-pencil sample, there were two other differences that may have impacted the level of interests.

First, the racial and ethnic minority representation was substantially larger in the 2005 GRS, increasing from 8\% in the 1994 Strong GRS to $32 \%$ for women and 24\% for men in the 2005 GRS. The 2005 GRS distribution for males matched the U.S. Census, whereas the female distribution overrepresented racial and ethic minorities. Second, the 2005 GRS was substantially less educated than the 1994 GRS. In the 2005 GRS, there were proportionally more people with technical training or some college and proportionally less people with professional or doctoral degrees. Users of the 2005 Strong may want to consider this information when interpreting the 2005 Strong. Future researchers may want to follow up in exploring the potential impact of a more diverse, less educated normative comparison group.

\section{Limitations}

Although this is the first study to compare the 1994 and 2005 parallel content scales, one limitation was the need to have students complete the items using the 2005 5-point item response format rather than the 1994 3-point item response format, although Donnay et al. (2005) provided evidence of the continuity of the 5-point scaling. This limitation was specifically addressed by Borgen, Larson, Bailey, Thompson, and Donnay (2005), who gave participants both the 1994 and the 2005 Strong testing booklets. They found similar results to these findings. Second, the number of racial and ethnic minorities in the sample was insufficient to generate separate means and correlations by ethnic group. Prior studies have not shown differences across ethnicity for the 1994 Strong (Harmon et al., 1994; Lattimore \& Borgen, 1999) but differences need to be examined in the 2005 Strong. 


\section{Recommendations for Future Research}

In this study, we provide comparison data of the 2005 Strong and its 1994 predecessor. The 2005 Strong is in its infancy, and as such, more work needs to be done demonstrating its reliability and validity in different populations. Gasser et al. (2007) as well as Donnay et al. (2005) have begun the process by presenting concurrent validity estimates. More work is needed to ascertain whether interests as measured with the 2005 Strong as opposed to the 1994 Strong are similarly related to self-efficacy (e.g., Rottinghaus, Larson, \& Borgen, 2003) and personality (Larson, Rottinghaus, \& Borgen, 2002; Staggs et al., 2007). Moreover, studies are needed that provide evidence of the 2005 Strong's usefulness in predicting important criteria like educational aspirations (Rottinghaus, Lindley, Green, \& Borgen, 2002), occupational membership (Donnay \& Borgen, 1996; Lattimore \& Borgen, 1999) and choice of major (Issacs, Borgen, Donnay, \& Hansen, 1997; Ralston, Borgen, Rottinghaus, \& Donnay, 2004).

\section{Clinical Usefulness}

The results of this study have several implications for counselors for whom use of the new 2005 Strong is encouraged with the following caution. Counselors may see higher scores overall with the 2005 Strong, especially for the Realistic and Investigative GOTs and the Mathematics and Teaching BISs for women. Moreover, higher scores may emerge with the Nature and Agriculture BIS for both sexes. Counselors should use caution in the direct interpretation of the 2005 Strong using 1994 interpretative strategies on the 2005 Nature/Agriculture BIS and the 2005 Risk Taking PSS because they have been altered in content.

As with the 1994 Strong, in general counselors may see males expressing more realistic interests (particularly mechanical and military interests) and may see females expressing somewhat more social interests. However, the observed mean sex differences between analogous scales of the 1994 and 2005 Strongs could have specific implications, especially for women. Because the observed mean sex differences on the content scales of the two versions resulted in women scoring higher on some of the 2005 BIS scales, their interest levels on some of the GOTs (particularly the Realistic GOT) could become inflated compared to how they would score on the 1994 Strong. Counselors will have to be cautious in how they interpret these higher scores as they may be more reflective of differences in the standardization samples.

\section{CONCLUSION}

This study provided an initial attempt at determining the equivalency of the 1994 and 2005 Strong Interest Inventories, and demonstrated the overall stability of the interpretive implications of the 2005 Strong. Additional research must be 
conducted on the 2005 Strong to further inform its users of the measure's reliability and validity. Though the results of this study serve as a springboard for the incremental increase in user acceptance of the revised 2005 Strong Interest Inventory, more work will be needed before many users' comfort threshold is reached.

\section{REFERENCES}

Betz, N., Borgen, F., Rottinghaus, P., Paulsen, A., Halper, C., \& Harmon, L. (2003). The Expanded Skills Confidence Inventory: Measuring basic dimensions of vocational activity. Journal of Vocational Behavior, 62, 76-100.

Borgen, F. H., Larson, L. M., Bailey, D. C., Thompson, R. C., \& Donnay, D. A. C. (2005). Continuity of the 1994 and 2004 Strong Interest Inventories. Unpublished manuscript.

Campbell, D. P., \& Borgen, F. H. (1999). Holland's theory and the development of interest inventories. Journal of Vocational Behavior, 55, 86-101.

Campbell, D. P., \& Holland, J. L. (1972). A merger in vocational interest research: Applying Holland's theory to Strong's data. Journal of Vocational behavior, 2, 353-376.

Cohen, J. (1988). Statistical power analysis for the behavioral sciences (2nd ed.). Hillsdale, NJ: Lawrence Earlbaum Associates.

Donnay, D. A. C., \& Borgen, F. H. (1996). Validity, structure, and content of the 1994 Strong Interest Inventory. Journal of Counseling Psychology, 43, 275-291.

Donnay, D. A. C., Thompson, R., Morris, M., \& Schaubhut, N. (2005). Strong Interest Inventory manual: Research, development, and strategies for interpretation. Palo Alto, CA: Consulting Psychologists Press.

Gasser, C. E., Larson, L. M., \& Borgen, F. H. (2007). Concurrent validity of the 2005 Strong Interest Inventory: An examination of gender and major field of study. Journal of Career Assessment, 15, 23-43.

Hansen, J. C. (1986). Strong Vocational Interest Blank/Strong-Campbell Interest Inventory. In W. B. Walsh \& S. H. Osipow (Eds.), Advances in vocational psychology (pp. 1-29). Hillsdale, NJ: Erlbaum.

Hansen, J. C. (1990). Interest inventories. In S. Goldstein \& M. Hersen (Eds.), Handbook of psychological assessment (pp. 173-194). Elmsford, NY: Pergamon Press.

Hansen, J. C. (1994). The measurement of vocational interests. In M. G. Rumsey \& J. H. Harris (Eds.), Personnel selection and classification (pp. 293-316). Hillsdale, NJ: Lawrence Erlbaum.

Hansen, J. C., \& Campbell, D. C. (1985). Manual for the Strong Interest Inventory (4th ed.). Palo Alto, CA: Stanford University Press.

Harmon, L. W., Hansen J. C., Borgen, F. H., \& Hammer, A. L. (1994). Applications and technical guide for the Strong Interest Inventory. Palo Alto, CA: Consulting Psychologists Press.

Holland, J. L. (1985). Making vocational choices (2nd ed.). Englewood Cliffs, NJ: Prentice-Hall.

Holland, J. L. (1997). Making vocational choices: A theory of vocational personalities and work environments (3rd ed.). Odessa, FL: Psychological Assessment Resources.

Isaacs, J., Borgen, F. H., Donnay, D. A. C., \& Hansen, T. A. (1997, August). Self-efficacy and interests: Relationships of Holland themes to college major. Poster session presented at the annual convention of the American Psychological Association, Chicago.

Larson, L., Rottinghaus, P., \& Borgen, F. H. (2002). Meta-analysis of Big Six interests and Big Five personality factors. Journal of Vocational Behavior, 61, 217-239.

Lattimore, R. R., \& Borgen, F. H. (1999). Validity of the 1994 Strong Interest Inventory with racial and ethic groups in the United States. Journal of Counseling Psychology, 46(2), 185-195.

Olsen, L. L. (1996). Concurrent validity of the 1994 Strong Interest Inventory: A comparison of criterion groups by gender. Unpublished doctoral dissertation, Iowa State University. 
Ralston, C. A., Borgen, F. H., Rottinghaus, P. J., \& Donnay, D. A. C. (2004). Specificity in interest measurement: Basic Interest Scales and major field of study. Journal of Vocational Behavior, 65, 203-216.

Rottinghaus, P. J., Larson, L. M., \& Borgen, F. H. (2003). The relation of self-efficacy and interests: A meta-analysis of 60 samples. Journal of Vocational Behavior, 62, 221-236.

Rottinghaus, P. J., Lindley, L. D., Green, M. A., \& Borgen, F. H. (2002). Educational aspirations: The contribution of self-efficacy, interests, and personality. Journal of Vocational Behavior, 61, 1-19.

Tellegen, A. (1982). Brief manual for the Differential Personality Questionnaire. Unpublished manuscript, University of Minnesota.

Tellegen, A. (2000). Manual for the Multidimensional Personality Questionnaire. Minneapolis, $\mathrm{MN}$ : University of Minnesota Press.

Staggs, G., Larson, L. M., \& Borgen, F. H. (2007). Convergence of interests and personality: Metaanalyses of the Multidimensional Personality Questionnaire and the Strong Interest Inventory. Journal of Career Assessment, 15, 423-445.

Strong, E. K., Jr. (1927). Vocational interest test. Educational Record, 8, 107-121. 